\title{
NEURO FUZZY CONTROL OF THE FES ASSISTED FREELY SWINGING LEG OF PARAPLEGIC SUBJECTS
}

\author{
Jaap H. van der Spek ${ }^{1}$, Wubbe J. R. Velthuis ${ }^{1}$, Peter H. Veltink ${ }^{1}$, Theo J. A. de Vries ${ }^{1}$ \\ ${ }^{1}$ Faculty of Electrical Engineering, University of Twente, P.O. Box 217, 7500 AE Enschede, the Netherlands, \\ mail to: J.H.vanderspek@student.utwente.nl
}

\begin{abstract}
We designed a neuro fuzzy control strategy for control of cyclical leg movements of paraplegic subjects. The cyclical leg movements were specified by three 'swing phase objectives', characteristic of natural human gait. The neuro fuzzy controller is a combination of a fuzzy logic controller and a neural network, which makes the controller self tuning and adaptive. Two experiments have been performed, in which the performance of the neuro fuzzy control strategy has been compared with a conventional PID control strategy.
\end{abstract}

\section{INTRODUCTION}

People who have suffered injury of the spinal cord often become paralyzed. Since the nerve pathways from the brain to the lower extremities are unrecoverably damaged they can no longer be controlled. Coordinated activation of muscles, i.e. stimulation of muscles, with the objective to restore muscle activity is called Functional Electrical Stimulation, FES.

In our case, we concentrate on the swing of the leg as can be specified by three 'swing phase objectives' [1].

hip angle range: the hip range, i.e. the 'stepsize' of the swinging leg, should reach a certain predefined constant value, similar to the 'stepsize' of human gait. Control of hip range is obtained by hip flexor stimulation, right after the beginning of the swing phase.

footclearance: in the forward swing of the leg, the knee has to be flexed to prevent the foot from touching the ground. Control of footclearance is done by stimulation of the hamstrings right before the beginning of the swing phase.

knee extension: at the end of the forward swing, the knee has to be fully extended to ensure safe body weight bearing. Control of knee extension is obtained by stimulation of the quadriceps at the end of the swing phase.

With this approach cross coupling effects of the various muscles are disregarded.

A conventional PID control strategy has previously been designed by $\mathrm{H}$. Franken [1] and tested experimentally. In this study, an alternative control approach was developed and was compared experimentally with the conventional approach.

\section{THEORY}

In analogy with PID control $[1,2]$, the neuro fuzzy approach was designed as a cycle-to-cycle controller [3]. Since the movement of the freely swinging leg is cyclical, it can be subdivided in separate cycles. In each cycle the swing phase objectives can be measured and compensated for.

The specified step response for the hip range was a trajectory given by a sigmoid function as a function of the cycle number, smoothly increasing the hip range to 0.8 [rad].
Due to system dynamics and inertia, 3 inputs were defined: the current hip range, $H R(k)$, the previous one, $H R(k-1)$, and the one before that, $H R(k-2)$, in which $k$ is the cycle number. Output was the adaptation of the burst time of the hip flexors.

The timing of the stimulation is shown in figure 1 , where the stimulation is triggered by the hip angle passing a certain threshold in the forward or backward swing:

a: wait time hamstrings

$b$ : wait time hip flexors

c: wait time quadriceps

$\phi_{\text {hip }}>0:$ hip flexion

$\phi_{\text {knee }}=0:$ extended knee

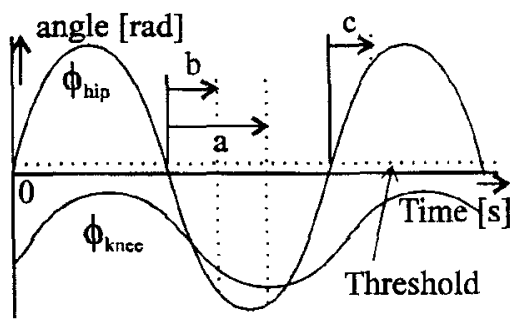

Fig. 1: Timing of the control actions

The hip range controller was implemented as a neuro fuzzy controller [3,4], as depicted in figures 2 and 3 :

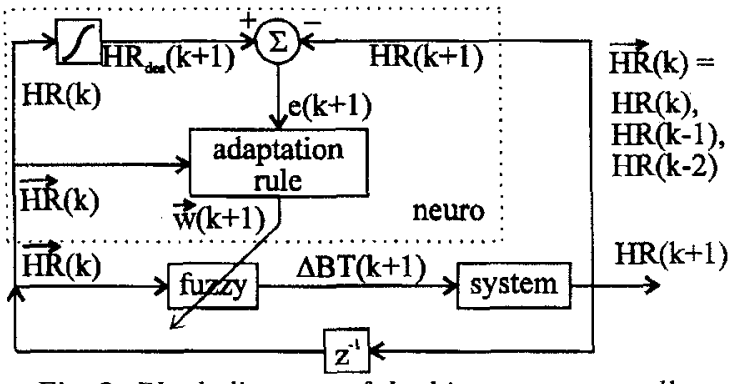

Fig. 2: Block diagram of the hip range controller

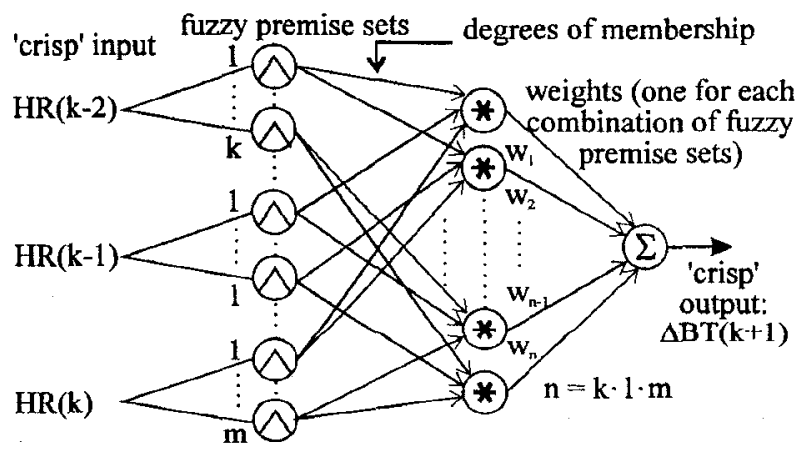

fig. 3: Neuro fuzzy controller in detail

From figure 2 it follows that the difference between the actual and desired hip range $e(k+1)$ is fed back to the neuro fuzzy controller. From $\mathrm{e}(\mathrm{k}+\mathbf{l})$ a desired burst time adaptation 
$\Delta \mathrm{BT}_{\text {des }}(\mathrm{k}+1)$ is derived which should be applied to the system if the same set of inputs $\mathrm{HR}(\mathrm{k})$ arises again.

From figures 2 and 3 , it can be seen that at the beginning of the cycle, $\Delta B T(k+1)$ is calculated based on the current set of weights $\vec{w}(k)$. At the end of the cycle, the new set of weights $\overrightarrow{\mathrm{w}}(\mathrm{k}+1)$ is calculated using a backpropagation learning rule with $H \vec{R}(k)$ as input and $\Delta B T_{\text {des }}(k+1)$ as output.

The controllers for knee extension and footclearance were implemented as purely fuzzy logic controllers and will not be considered in this paper.

\section{METHODS}

Although the footclearance and knee extension controllers werc closed loop fuzzy logic controllers, the hamstrings and quadriceps were controlled open loop in the fuzzy controlled experiments, to make a valid comparison with the PID control strategy, in which this was also done.

Two experiments have been performed until now. The subjects were placed in a standing frame, wearing an orthosis to restrict motion in the saggital plane. One leg was placed on a block, enabling the other leg to move freely. After the warming up exercise the controllers had to be tuned.

PID tuning was done by examining a passive swing of the leg. It was shown [2] that subsequent maximum hip angles are a measure for the damping of the system. The poles and zeros of the controller could be derived from this. The wait times for the various muscles ( $a, b$ and $c$, see figure 1) could also be derived from the passive swing. The gain was determined by repeatedly changing the gain until a satisfactory step response with tolerable overshoot was achieved.

The neuro fuzzy tuning was done by repeated tuning trials until the trajectory of the hip range was learned. Simulation studies [3] showed, that $10-15$ short stimulation trials would be sufficient to tune the controller.

The performance of both control strategies was tested in long fatigue trials of \pm 150 cycles, testing the ability of both control strategies to compensate for muscle fatigue.

\section{PRELIMINARY RESULTS}

Figure 4 shows preliminary results of the fatigue trials for the second subject. With the first subject no fatigue trials were performed due to time limitations. The first plot gives the hip range and the burst time of the hip flexors using the neuro fuzzy control strategy, both plotted against the cycle number. The second plot gives the same parameters plotted against the cycle number for the PID controller.

\section{DISCUSSION}

From figure 4 it can be seen that the neuro fuzzy as well as the PID controller are capable of compensating fatigue: both controllers seem to be reasonably well capable of controlling the hip range, until the maximum burst time (T_burst) of 600 [ms] was reached. The apparent quicker occurring fatigue in the fuzzy controlled experiment is not caused by worse control performance, but by the fact that a few additional tuning trials were needed prior to the neuro fuzzy experiment, while prior to the PID experiment sufficient rest was taken and only 2 tuning trials were performed. However in the PID experiment, larger stimulation amplitudes were used.

From the first plot, which was obtained after 5 tuning trials, it can be seen that the tuning of the neuro fuzzy controller went well: there is no clear difference in the hip range in the PID and neuro fuzzy experiments. The footclearance and knee extension were not plotted here, because in both control strategies, they were not controlled.
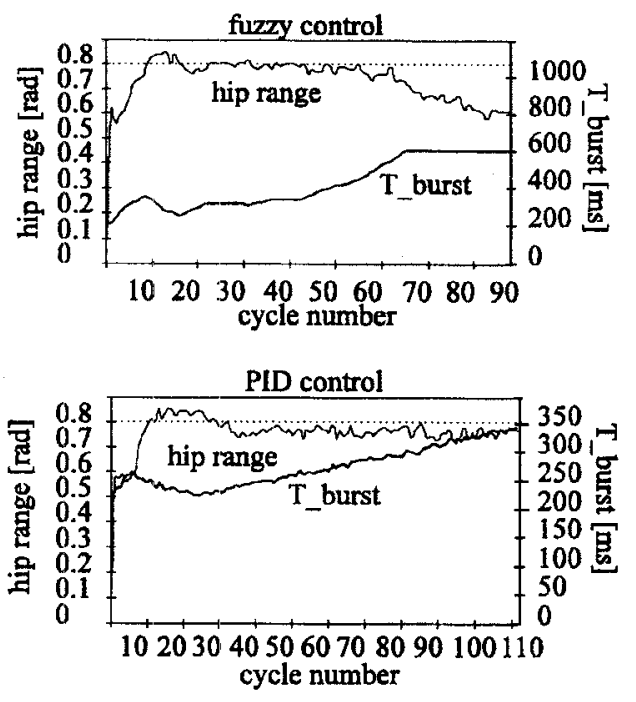

Fig. 4: Preliminary results, second subject

In future experiments neuro fuzzy controlled and PID controlled hip range performance will be further compared. Also, neuro fuzzy control combined with closed loop fuzzy logic trials of hamstrings and quadriceps stimulation will be performed to see whether this will significantly improve the footclearance and knee extension.

\section{REFERENCES}

[1] Franken, H.M., P.H. Veltink, G. Baardman, R.A. Redmeijer and H.B.K. Boom, 'Cycle-to-cycle control of swing phase of paraplegic gait induced by surface electrical stimulation', Med. \& Biol. Eng. \& Comput., vol. 33, pp. $440-451,1995$.

[2] P. H. Veltink, 'Control of FES induced cyclical movements of the lower leg', Med. \& Biol. Eng. \& Comput., North Sea special feature, vol. 29, pp. NS8-NS12.

[3] De Vries, T.J.A., W.J.R. Velthuis, J. van Amerongen and P.H. Veltink, 'Fuzzy and learning control of FES induced gait', IFAC conference 1996, San Fransisco, accepted.

[4] Lee, C.C. , 'Fuzzy logic in control systems: fuzzy logic controller', part I \& II, IEEE Trans. Sys., Man \& Cybern., vol. 20 , pp. $404-435,1990$. 